

\title{
Competencias y funciones profesionales: un análisis aplicado a la titulación de trabajo social en la universidad de la Rioja.'
} Professional competences and functions: an analysis applied to the social work degree at the university of la Rioja

\author{
Domingo Carbonero Muñoz ${ }^{*}$,Ana Belén Cuesta Ruiz Clavijo ${ }^{* *}$, Neus Caparrós Civera ***, Cecilia Serrano-Martinez ${ }^{* * * *}$ \\ * Universidad de La Rioja. Domingo.carbonero@unirioja.es; ** Universidad de La Rioja. ana-belen.cuesta@unirioja.es \\ *** Universidad de La Rioja. caparros@unirioja,es; **** Universidad de La Rioja. cecilia.serrano@unirioja.es
}

\begin{abstract}
:
This paper addresses the professional skills in the Social Work degree and the professional's role and the main domains of Social Intervention defined by ANECA (2004). The opinion in Social Worker is studied by an exploratory survey in La Rioja. In summary, it was collected 70 cases of Social Workers in professional. At the same time, it was developed a qualitative methodology by three groups of discussion formed by Social Worker and 32 groups of reflexions by students on Social Work. The active learnership or the social intervention practices are indicated such a relevant finding by the professionals and the students. At the end, the results are incorporated in the changes of the degree. Specifically, by recommendations in the Social Work degree.
\end{abstract}

Keywords: Social Work in education, significant learnership, innovation teacher.

\section{Resumen:}

El artículo aborda las competencias profesionales, las funciones definidas por la ANECA (2004) y los ámbitos de intervención en Trabajo Social. Mediante una encuesta exploratoria en La Rioja se estudia la opinión profesional de 70 trabajadores sociales en activo. Paralelamente, se desarrolla una metodología de investigación cualitativa mediante tres grupos de discusión formados por profesionales y 32 grupos de reflexión integrados por alumnos de grado en Trabajo Social. Las principales discusiones desde el ámbito profesional y de los estudiantes, se sitúan en torno a la metodología de aprendizaje activo o las prácticas de intervención profesional. Finalmente, los resultados obtenidos se incorporan en forma de recomendaciones en la reforma del título de grado.

\footnotetext{
1 Este artículo se ha desarrollado dentro del Proyecto de Innovación Docente titulado "El perfil del trabajador social en la sociedad actual", dentro de La Universidad de La Rioja" (Programa de Formación del Personal Docente e Investigador 2017/2018).
} 
Palabras clave: Trabajo social en educación, aprendizaje significativo, ética, innovación docente.

\section{Article info:}

Received: 06/03/2020 Received in revised form: 18/06/2020

Accepted: 25/06/2020 / Published online: 01/06/2020

DOI: http://dx.doi.org/10.5944/comunitania.20.5

\section{Introducción}

El debate se sitúa en torno a la dificultad de afrontar las competencias profesionales como medio para la inserción profesional o por su importancia en cuanto a finalidad y compromiso con el desarrollo futuro de la profesión (Cortina, 2000). Esta autora destaca aspectos claves tales como la generación de una comunidad profesional con un estatus reconocido o la generación de un cuerpo compartido como profesión.

El debate en torno a las competencias profesionales adquiere un carácter epistemológico en la medida que lleva a reflexionar sobre el objeto delTrabajo Social y su dimensión científica (Restrepo, 2002). A este respecto, se señala el carácter empírico y cotidiano de esta disciplina, incluyendo el trabajo con distintos sectores de población, elaborando propuestas, para hacer frente a los cambios permanentes.

En esta línea, se han desarrollado proposiciones de carácter reflexivo en cuanto a la adquisición de competencias formativas por parte del alumnado (Anguiano y Plasencia, 2007). Carácter que trata de despertar su carácter crítico entre alumnado y el mundo profesional. Se trata de conectar la teoría y la práctica en el aprendizaje, o en otras palabras, la necesidad de que los estudiantes sean conscientes de sus fortalezas y de sus debilidades (Anguiano y Plasencia, 2007).

Existen estudios realizados con profesionales y con alumnos, para identificar sus necesidades, e incorporarlas como parte de un proceso participativo (Del Rincón, 2007). Otros estudios, han servido para dar contenido y sentido al desarrollo de las competencias por parte de los estudiantes (Ramos-Feijóo, C. Ariño, M., Berasaluze, A. Dellavalle, M. et. al. 2013; Ramos Feijóo, C. Lorenzo García, J. y Valíllo Pertusa, P., 2013).

Monreal y Terrón (2012) señalan que esta perspectiva crítica debe vincularse con la perspectiva del profesorado. Principalmente, la realización de actividades conjuntas y compartidas lleva a romper con el individualismo en la planificación del profesorado o a la posibilidad de fomentar una visión integral y conjunta en la resolución de los problemas. Aunque este tipo de análisis no se ha llevado a cabo en este artículo, si se apuntan avances en la realización de actividades conjuntas mediante diseños de proyectos de innovación docente o la elaboración de materiales interdisciplinares. 
La primera parte del artículo aborda la formación en competencias y los cambios que provocó la incorporación al Espacio Europeo de Educación Superior (EEES) (Méndez, 2004; Gil, 2004; Vázquez, 2011; Ramos-Feijóo, Ariño, Berasaluze, et. al., 2013; Ramos Feijóo, Lorenzo, y Valíllo, 2013). Mediante una revisión bibliográfica a través de Dialnet, se ha profundizado en la definición de competencias en Trabajo Social, incluyendo planteamientos que hacen referencia a la diferencia entre competencias generales o específicas, o la aplicación a campos específicos como son las prácticas curriculares, la focalización en los niveles de intervención o en sectores específicos.

En el resto del artículo se presentan las secuencias y los hallazgos más relevantes respecto del Grado en Trabajo Social en la Universidad de La Rioja, en concreto sobre objetivos generales y específicos. Principalmente, se hace referencia a aspectos vinculados a la composición y la estructura del grado, a las competencias y funciones profesionales que necesitan ser incorporadas o reforzadas, la visión del alumnado sobre su proceso formativo y la incorporación de cuestiones vinculadas a la ética profesional.

En el apartado de metodología se diferencian dos fases. La primera de éstas profundiza en la visión del alumnado mediante grupos de reflexión y de discusión, sirviendo para indagar en la perspectiva crítica y constructivista anteriormente señalada. La segunda de las fases, mediante una encuesta, de corte exploratorio, se indaga en las percepciones profesionales sobre las competencias que deben de adquirirse y reforzar por el alumnado en su proceso de formación. Retomando las propuestas de Cortina (2000) y de Restrepo (2002), la legitimación del objeto del Trabajo Social viene respaldada por la conexión con el mundo social y profesional.

Este artículo persigue una sistematización en la titulación de Trabajo Social en la Universidad de La Rioja, con el fin de presentar una información contrastable y útil en el proceso de modificación del título. Para ello, se han revisado las siguientes temáticas: estudios de Trabajo Social en España y competencias adquiridas en Trabajo Social. El resto del capítulo se ha estructurado en metodología, resultados procedentes de una estrategia cualitativa y cuantitativa de investigación realizada con alumnos y Trabajadores Sociales en activo, respectivamente. Tal y como señalan Anguiano y Plasencia (2007), se incorporan tanto "saberes prácticos" como "técnicos", vinculando la adquisición de competencias formativas al desempeño de habilidades o de capacidades profesionales. Una última parte en la cual se presentan propuestas a considerar en la modificación del grado.

\section{Estado de la cuestión I: La formación en competencias en los estudios de Trabajo Social}

Previo al proceso de Bolonia, los debates en torno a los planes de estudios venían condicionados por el aislamiento de la profesión deTrabajo Social en el contexto internacional y un retraso en el desarrollo del Estado de Bienestar en España (Méndez-Boni- 
to, 2004). Esta situación ha condicionado durante el final del siglo XX y comienzos del $\mathrm{XXI}$, que se afianzase, el desarrollo delTrabajo Social como disciplina. En la mayoría de las ocasiones, los debates más repetidos durante la última década de los 90 y comienzos del siglo XXI están relacionados con las etapas por las que transcurren los estudios de formación en Trabajo Social en distintas universidades españolas (Martínez, 2018; Barranco, 2011). Los problemas en la literatura inciden en la confusión entre Trabajo Social y Servicios Sociales (Ariño, 1998), la importancia de clarificar el objeto del Trabajo Social (Zamanillo y Gaitán, 1991; Zamanillo, 1992), la conexión de los planes de estudios con las diferentes prácticas profesionales (Méndez, 1987) o la respuesta a los cambios sociales y la emergencia del tercer sector (Aguiar, 2006).

Las competencias profesionales en los grados de Trabajo Social adquieren su importancia en el proceso de convergencia europea universitaria. El asumir un enfoque basado en competencias, se inspira tanto en articulación entre necesidades sociales y educación, como en la adaptación a entornos laborales concretos. Por un lado, se identifican nuevos desafíos vinculados a las transformaciones sociales, y por otro, el desarrollo de las competencias se vincula a la capacidad de adaptarse a nuevas exigencias y a nuevos contextos laborales (Gómez, 2010).

Tras la revisión bibliográfica efectuada y recogida en la tabla 1, se muestran cuatro perspectivas desde las que indagar en las competencias en Trabajo Social: desempeño profesional, cuestiones éticas, competencias profesionales para afrontar funciones profesionales en campos especializados de intervención y competencias profesionales aplicadas a las prácticas o a aspectos metodológicos de intervención social. Otros aspectos hacen referencia tanto a las competencias investigadoras o al grado de satisfacción con la titulación (Leitao, Álvarez y Barroso, 2018; Medina y Blanco, 2014). Estas cuestiones se han vinculado a la inserción profesional de los egresados. Sin embargo, si se han considerado complementarias y útiles en el estudio de las competencias formativas.

1. Competencias y desempeño profesionales. Las competencias formativas en el campo de la educación persiguen la promoción de habilidades y destrezas entre el alumnado con la finalidad de lograr su adecuación al contexto profesional (Gómez, 2010). Si bien, Vázquez (2011) destaca las competencias profesionales como un paso necesario en la formación del alumnado para afrontar la incorporación al mercado laboral.

Distintos autores han debatido sobre el tipo de competencias que son necesarias para lograr esta adaptación al mundo profesional. Por un lado, Moñivas (2004) ha diferenciado entre competencias generales o competencias especializadas ${ }^{2}$

2 El modelo generalista hace referencia a una amplia gama de competencias comunes a diferentes campos de prácticas, mientras que la especialización en competencias formativas queda vinculada a campos de prácticas específicos. 
adaptadas a cada campo de prácticas. Por otro lado, Vázquez et. al (2011) distingue tres tipos de competencias: 1. Competencias generales. 2. Específicas $^{3}$; 3. Profesionales4; Ramos-Feijó, et. al (2013) promueven, en un marco comparativo, la evaluación de las competencias por parte del alumnado, distinguiendo competencias promovidas, adquiridas, practicadas y evaluadas ${ }^{5}$.

2. Las cuestiones éticas son competencias necesarias en el trabajo con personas en situación de dificultad. Aguayo (2004) y Cortina (2000) hacen referencia a obligaciones morales de obligado cumplimiento que nos legitiman ante el conjunto de la sociedad. A este respecto, las competencias no sólo son un medio que se plantea para la inserción profesional, sino que está orientado a la finalidad de la profesión. Además, emergen cuestiones tales como los mínimos éticos que deben de defenderse en la intervención profesional, las actitudes y aptitudes que mostramos o nuestra idoneidad profesional ante una intervención social.

3. Las competencias profesionales se han planteado con la finalidad de preparar a los estudiantes en el desempeño de funciones profesionales y de campos especializados de la intervención social. Podrían existir tantos estudios como funciones y ámbitos de trabajo, sin embargo, algunos de los trabajos destacados en la revisión bibliográfica, han hecho referencia a competencias generales y específicas, vinculadas a los ámbitos de interculturalidad, salud, Tercer Sector de Acción Social o género.

4. Competencias profesionales aplicadas a las prácticas externas o a aspectos metodológicos de intervención social. Un primer diseño en las competencias específicas se realiza en las asignaturas deTrabajo Social con personas y familias, Trabajo Social con grupos y de Trabajo Social con comunidades. Su evaluación se realiza mediante tres vías de aplicación: los contenidos de dichas asignaturas; la conexión con la asignatura de Habilidades profesionales del

3 La distinción realizada por Váquez et. als (2011) sitúa a aquellas competencias generales o transversales, como competencias comunes a todas las titulaciones universitarias. Estas competencias generales pueden ser de tres tipos: instrumentales o desatinadas a ser empleadas como herramientas para conseguir algo; interpersonales, destinadas a favorecer la relación y la interacción social; y sistémicas. Estas últimas competencias fomentan la capacidad de abordar por parte del alumnado distintos escenarios profesionales durante su trayectoria laboral

4 Las competencias profesionales incluyen una serie de rasgos vinculados a las actitudes, valores, grado de éxito o fracaso en el ejercicio del rol profesional, la capacidad de afrontar situaciones problemáticas o de adaptarse y enfrentarse a dificultades distintas a las inicialmente provistas. Además, ha de tenerse en cuenta la posibilidad de entrenar la adquisición de competencias en el transcurso de su vida laboral.

5 Se promueve la reflexión entre el alumnado respecto de su aprendizaje. Principalmente, se trata de contrastar la visión del aprendizaje por parte del alumnado con la visión de la enseñanza por parte del profesorado. Para ello, se persigue que los propios estudiantes sean capaces de conocer su adquisición, práctica o desempeño profesional y si han sido evaluadas en el proceso formativo. 
Trabajo Social; y la aplicación de las competencias en las prácticas y en algunas guías docentes

Gómez del Toro y Fernández (2016) tratan de elaborar propuestas orientadas a la modificación del título. Vinculan la adquisición de las competencias con la satisfacción percibida entre el alumnado con la titulación. Para ello, estos autores incluyen cinco bloques de competencias centradas en cuestiones metodológicas y Trabajo Social:

1) análisis y diagnóstico;

2) diseño y planificación;

3) desarrollo y ejecución de programas;

4) evaluación y seguimiento;

5) propuestas de mejora.

La evaluación en la adquisición de competencias se mide en las materias vinculadas a las prácticas externas y de trabajo de fin de grado. El análisis de competencias realizado por Pastor (et. al. 2016) evalúa la adquisición de las competencias específicas para las asignaturas de personas, familias, grupos, organizaciones y comunidades. Para ello, en el marco de las asignaturas de las prácticas externas de la titulación se integran competencias transversales de otras titulaciones y específicas del grado de Trabajo Social, así como competencias genéricas derivadas del desempeño de la práctica profesional. 


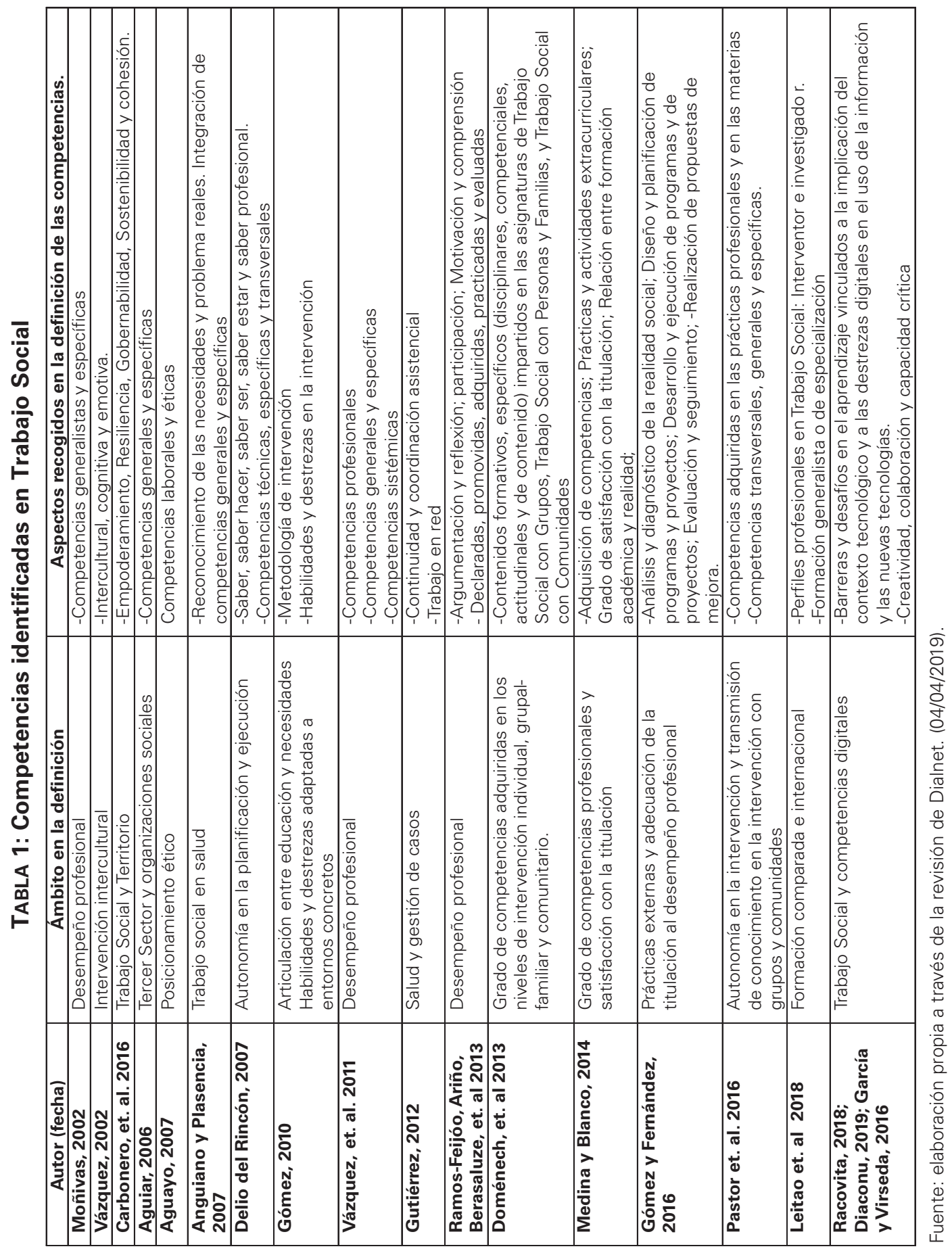

Comunitania: International Journal of Social Work and Social Sciences № 20 / July 2020 


\section{Estado de la Cuestión II: Funciones y ámbitos de intervención en Trabajo Social}

La Agencia Nacional de Evaluación de la Calidad y Acreditación (ANECA), en el Libro Blanco delTítulo de Grado en Trabajo Social (ANECA, 2004), define el perfil profesional del Trabajo Social en el que se encuentran las competencias mencionadas en el apartado anterior y las funciones realizadas por los profesionales:

- Asistencial

- Preventiva

- Promocional y educativa

- Mediación

- Transformadora

- Planificación-evaluación

- Rehabilitadora.

- Gerencia y administración

- Investigación y docencia.

No obstante, estas funciones habrán de adaptarse a cada ámbito de intervención. De esta manera, las funciones específicas en cada ámbito de aplicación serán aqueIlas que resulten de adaptar las funciones genéricas al sistema de protección desde el que se ejerce la intervención y al equipo profesional del que se forma parte. En consecuencia, el perfil profesional se desarrolla en los siguientes ámbitos de intervención (ANECA, 2004: 140).

a) Servicios Sociales. En el primer y segundo nivel de intervención del sistema público se articulan funciones profesionales vinculadas a la aplicación de prestaciones económicas y servicios. En un primer caso los servicios sociales de primer nivel, que están dirigidos a toda la población, prestan una atención básica y general. Por otra parte, los servicios sociales de segundo nivel, especializados atienden a diversos colectivos tales como personas sin hogar, personas mayores, infancia y familia, mujer, personas con discapacidad, inmigrantes y refugiados (Anaut, 2019).

b) Salud. La intervención social sanitaria se desempeña en varios niveles: atención primaria y especializada. La atención primaria está ubicada en los centros de atención primaria o de salud, y también especializada en recursos hospitalarios. En espacios sociosanitarios tales como centros de convalecencia, cuidados paliativos y también en la atención a problemas de salud mental, urgencias u otros ámbitos (mutuas laborales) (Masfret, 2008). Sus funciones se vinculan mediante el desempeño de la intervención psicosocial, la atención de problemas no sanitarios, la gestión de la cartera de servicios en salud y la pre- 
paración del alta de pacientes e intervención familiar o la comunidad. (Masfret, 2008; Ituarte, 2009).

c) Educación. En el marco de la actuación educativa se encuentra en los Equipos de Orientación Educativa y psicopedagógica (EOEPS) de los centros y las zonas de actuación. Su intervención abarca los programas de atención a la diversidad, y en menor proporción, existen trabajadores sociales en colegios e institutos.

Dentro de los EOEPS los Trabajadores Sociales ejercen funciones psicopedagógicas y sociales. Las acciones psicopedagógicas incluyen la colaboración en la adquisición de competencias comunicativas y de aprendizaje, el nexo con la familia, la colaboración en actividades extraescolares, la intervención en planes de convivencia vinculados a la resolución de conflictos y la escolarización. En segundo lugar, el marco social se incluye las acciones dirigidas a evitar el absentismo, a integrar a alumnos con problemas de adaptación y a realizar el seguimiento y control del absentismo, coordinándose con equipos de trabajo del entorno.

d) Justicia. En este ámbito, la profesión del Trabajo Social desempeña su intervención en diversos espacios profesionales. Se pueden destacar los siguientes: integrante de los equipos psicosociales en los juzgados de primera instancia (Familia), el punto de encuentro familiar, los programas de mediación intra judicial y extrajudicial, las clínicas médico-forenses, el instituto anatómico forense, los decanatos o juzgados de primera instancia e instrucción o las oficinas de atención a la víctima (Ruiz, 2013). En el marco de intervención con menores desarrollan sus acciones en acogimiento familiar y residencial, en intervenciones con menores infractores y libertad vigilada. Finalmente, se recogen funciones específicas en el marco de las instituciones penitenciarias (unidad de medidas y penas alternativas y de servicios sociales penitenciarios) (Secretaría de Instituciones Penitenciarias, 2018).

En la mayoría de estos ámbitos, se cumplen aspectos vinculados a procedimientos de guarda y custodia de menores en procesos de separación y divorcio o la regulación del régimen de visitas. Al mismo tiempo, se realizan informes periciales sobre causas o a cuestiones penales. Finalmente, la intervención penitenciaria se relaciona con el seguimiento de libertades vigiladas, alternativas de prisión o de trabajos para la comunidad (Ruiz, 2003).

e) Empresa. En las empresas y las organizaciones se sitúan intervenciones vinculadas a empresas, a empresas de servicios sociales y educativas, en empresas de inserción social, gabinetes y consultorías (Raya y Caparrós, 2013). EI desempeño de las acciones vinculadas a grandes empresas, se encuentra el desarrollo de la Responsabilidad Social Empresarial con fines externos e internos. Los ejemplos hacen referencia a los programas relativos a adecuación de la política de recursos humanos, atención a problemas de salud, clima laboral o la gestión de la política social de la empresa. Asimismo, las funciones en 
gabinetes privados pueden vincularse acciones de Trabajo Social Individual, propuestas de investigación y desarrollo de acciones individuales en entidades sociales, las acciones en empresas de inclusión social hacia colectivos con dificultades especiales o el desarrollo de los programas de formación tanto a usuarios como a profesionales.

f) Tercer Sector de Acción Social. En organizaciones y entidades sin ánimo de lucro, que trabajan con programas preventivos, asistenciales, formativos o de inserción social y laboral. Entre otras actividades se encuentran:

1) el apoyo y el asesoramiento en la organización;

2) la posibilidad de facilitar información;

3) la participación y coordinación en equipos interdisciplinares;

4) la gestión de actividades;

5) la tramitación de ayudas y de prestaciones;

6) la planificación, gestión y ejecución de proyectos y prestaciones

En la revisión teórica se han incluido competencias vinculadas a otros campos tales como el digital o de territorio. Éstas no aparecen en el campo de competencias de la ANECA. Consideramos que sería necesario incluirlas para reflejar una mayor variedad de competencias y, de este modo, actualizar el listado existente.

\section{Objetivos}

\subsection{Objetivos generales}

1. Analizar la visión del alumnado y de los profesionales en activo en cuanto al proceso formativo del grado en Trabajo Social y de aquellos aspectos a incorporar en los planes de estudios.

\subsection{Objetivos específicos}

1. Conocer la visión del alumnado en cuanto a contenidos, asignaturas, estructura del grado $u$ otros recursos vinculados a intercambios, ayudas u orientación profesional.

2. Explorar la visión profesional de los trabajadores sociales en activo, sobre funciones profesionales, competencias formativas, dilemas éticos y consolidación delTrabajo Social según sectores de intervención. 
3. Vincular las reformas del plan de estudios a las fortalezas y debilidades percibidas por el alumnado de la titulación.

\section{Metodología}

La estrategia cualitativa se caracteriza por su flexibilidad en el diseño, la elección de casos representativos y una reflexión constante durante el proceso de investigación que se traduce en la incorporación de propuestas (Ruiz de Olabuénaga, 1989). Mediante la elaboración de "grupos de reflexión" y técnicas de intervención grupal se indaga en la percepción del alumnado respecto del grado. Por otro lado, mediante una segunda estrategia de investigación, de corte cuantitativo, se ha explorado la percepción del mundo profesional en relación a las funciones del Trabajo Social, su consolidación por ámbitos y los cambios o ajustes profesionales en los roles y funciones en los diversos ámbitos profesionales.

El diagnóstico en torno al plan de estudios del grado de Trabajo Social se ha desarrollado en las siguientes secuencias:

1.Uso de Dialnet. Revisión las publicaciones acerca de las siguientes temáticas: estudios de Trabajo Social en España y competencias del Grado.

2. Diseño de una estrategia cualitativa dirigida al alumnado para identificar los temas de interés, un guion adaptado a la discusión, la formación y la ejecución de grupos.

3. En paralelo al punto anterior, se procedió a la realización de una encuesta dirigida al mundo profesional y con fines exploratorios. En esta fase se confeccionó un cuestionario semiestructurado y la realización de pruebas pretest. Finalmente, se obtuvieron 70 cuestionarios de trabajadores sociales de diferentes ámbitos de intervención.

4. Redacción de la memoria de la investigación, en la que se incorporaron posibles recomendaciones en materia de plan de estudios.

\subsection{Primera fase del diagnóstico: Percepción del grado de Trabajo Social entre el alumnado}

5.1.1 Grupos de reflexión, técnica grupal y elementos de análisis. Se llevaron a cabo 32 grupos de reflexión integrados por 5-6 alumnos de los cuatro cursos del grado. La finalidad era promover la reflexión acerca de las cuestiones planteadas. Para ello, se intervino de forma "semi-conducida" por parte del profesorado, con el fin de presentar la dinámica y que todas las temáticas fueran abordadas por el alumnado. Sin embargo, se consideró su rol no directivo con el propósito de no condicionar los 
debates iniciados por el alumnado. Para ello, una vez explicada la dinámica, el profesor se mantuvo al margen de las discusiones en los grupos, y en otros casos, directamente salió del aula (Serrano-Martínez, et. al. 2019).

Se persigue generar un espacio de reflexión en los estudiantes sobre el perfil profesional, los aprendizajes y su formación. Para ello, se abordaron cuestiones vinculadas al contenido, las asignaturas y a otros aspectos de la titulación. Mediante la formulación de preguntas abiertas, se identificaron fortalezas y debilidades percibidas por el alumnado. Los temas abordados se recogen en el cuadro 1.

\section{CUADRO 1: Guión de trabajo}

- ¿Qué habéis aprendido? ¿Qué habéis echado de menos?

- Contenido

- Asignaturas

- Estructura/organización

- Profesorado

- Servicios administrativos/gestión

- Información sobre: intercambios, becas, ayuda, otros estudios...

- ¿En qué os sentís mejor preparados?

- ¿En qué os sentís peor preparados?

- Observaciones

Fuente: Elaboración propia.

5.1.2. Características de la muestra cualitativa. Se realizaron 32 grupos de reflexión en los cuatro cursos del grado de Trabajo Social de la Universidad de La Rioja. Cada uno de estos grupos estuvo compuesto por 5 o 6 personas. Se alcanzaron 170 alumnos, un $85 \%$ del total de los estudiantes que componen el grado. A grandes rasgos, la edad media es de 20 años y de mayoritariamente de género femenino. La duración aproximada fue de una hora y media.

\subsection{Segunda fase del diagnóstico: percepciones del grado de Trabajo Social en el ámbito profesional}

El enfoque cuantitativo aborda la medición de opiniones o de actitudes, además de buscar relaciones de causalidad entre los fenómenos estudiados (Cea D'Ancona, 2001). Se procedió a la elaboración de una encuesta con la finalidad de recoger la opinión del mundo profesional respecto de las funciones del Trabajo Social y su consolidación en distintos ámbitos o sectores de intervención social. En esta 
línea, se pueden identificar estudios específicos vinculados a la construcción de escalas formativas y al grado de conocimiento de las competencias (Medina y Blanco, 2014).

Para ello, se realizó un muestreo de conveniencia, utilizando sólo aquella parte de la muestra accesible por parte del equipo de investigación. Esta parte del trabajo tuvo un carácter exploratorio, debido a que no se elaboró un marco poblacional desde el cual analizar extraer una muestra representativa del conjunto de los trabajadores sociales. Si bien, su empleo es útil en un momento inicial del trabajo o para sugerir posibles vías de investigación (Sabater, 2015). En la recogida de la información colaboraron distintas universidades pertenecientes al Campus Iberus (La Rioja, Lleida o Pública de Navarra) y el Colegio Oficial de Diplomados en Trabajo Social y Asistentes Sociales de La Rioja. (CODTAAS) En la elaboración del cuestionario se introdujeron 6 apartados mediante los cuales se abordaron los aspectos identificados en el cuadro 2.

\section{CUADRO 2: Temáticas abordadas en el cuestionario}

- Definición del Trabajo Social. Para ello, se abordó la definición de Melbourne (FITS, 2014)

- Grado de acuerdo en torno a funciones del trabajador social. Entre ellas, se encuentran las siguientes:

- Identificación de funciones desempeñadas en la profesión que no son identificadas como específicas de Trabajo Social.

- Nivel de acuerdo y desacuerdo respecto del grado en que otras profesiones realizan funciones que desempeñan trabajadores sociales.

- Identificación y grado de importancia de las competencias profesionales que deben de adquirirse y obstáculos que existen en la formación del alumnado.

- Grado de consolidación delTrabajo Social en distintos ámbitos profesionales.

- Identificación de dilemas éticos

- Introducción de cambios en la práctica profesional, y precisión de dichos cambios.

Fuente: Elaboración propia.

También se emplearon distintos estadísticos en el análisis efectuado (media y la desviación típica) mediante el programa SPSS V. 23. 


\subsubsection{Grupo de discusión con profesionales}

Se realizaron tres grupos de discusión mixtos integrados por profesionales de entidades sociales, estudiantes y docentes del grado de Trabajo Social de la universidad de La Rioja. Mediante la metodología de grupos de discusión formulada entre otros por lbáñez (1985), se trató de alcanzar discursos consensuados a nivel grupal. Los grupos se estructuraron en torno a los sectores de población de infancia, dependencia y personas mayores. En el primer caso los grupos se realizaron en el marco de una jornada de infancia organizada por la Universidad de La Rioja, en la que se abordaron dilemas, retos y estrategias de la disciplina del Trabajo Social. Dicha jornada se realizó el 7/3/18. Participaron 22 personas que fueron moderadas por profesoras del área de Trabajo Social.

El grupo de personas mayores y personas en situación de dependencia tuvo lugar el 14/6/18 en la sede del CODTSAS de La Rioja. Participaron 9 personas de las cuales 4 eran profesionales de entidades de personas mayores, 3 de entidades dirigidas a personas con discapacidad, un representante del CODTAAS y una profesora del grado que moderó el grupo.

Los ejes conductores de los grupos fueron la identificación de estrategias de intervención, dilemas y retos relativos a la práctica profesional relacionados con las siguientes cuestiones:

1. Funciones de los trabajadores sociales.

2. Percepción de este profesional por los usuarios y otros profesionales

3. Intrusismo profesional

4. Necesidades detectadas

5. Otras

\section{Discusión: Percepciones en torno el grado en Trabajo Social: discurso profesional y del alumnado}

\subsection{Consenso y debate en torno a las funciones del Trabajo Social. Encuesta y grupos de discusión}

Las funciones de información y de diagnóstico son aquellas que más consenso han alcanzado entre los profesionales encuestados, situándose sus respuestas en los mayores niveles de acuerdo. Asimismo, estas funciones superan el $70 \%$ y son aquellas con menores niveles de desviación típica. En segundo lugar, otras funciones que han tenido un consenso muy favorable hacen referencia a las funciones de planificación, atención directa, fomento de la integración y coordinación. Estas fun- 
ciones alcanzan el $60 \%$ en opinión muy favorable. Sin embargo, aquellas como planificación y coordinación son las funciones con mayores niveles de desviación típica, alcanzando .86 y .93 . (Tabla 2 )

TABLA 2: Grado de acuerdo y desacuerdo en las funciones desempeñadas por parte de los Trabajadores Sociales

\begin{tabular}{|c|c|c|c|c|c|c|c|c|}
\hline & 1 & 2 & 3 & 4 & 5 & $\mathbf{N}$ & Media & $\begin{array}{l}\text { Desviación } \\
\text { típica }\end{array}$ \\
\hline Información & 0 & 0 & 7.9 & 20.6 & 71.4 & 69 & 4.63 & .63 \\
\hline Diagnóstico & 0 & 0 & 9.5 & 11.1 & 79.4 & 69 & 4.69 & .63 \\
\hline Prevención & 0 & 3.2 & 15.9 & 22.2 & 58.7 & 69 & 4.36 & .86 \\
\hline Planificación & 0 & 0 & 4.8 & 34.9 & 60.3 & 69 & 4.55 & .58 \\
\hline Atención directa & 0 & 3.2 & 6.3 & 22.2 & 68.3 & 69 & 4.55 & .75 \\
\hline Supervisión & 0 & 9.5 & 19 & 30.2 & 41.3 & 69 & 4.03 & .99 \\
\hline $\begin{array}{l}\text { Promoción de la creación } \\
\text { desarrollo y mejora }\end{array}$ & 0 & 3.2 & 14.3 & 28.6 & 54 & 69 & 4.33 & .84 \\
\hline Fomento de la integración & 0 & 1.6 & 4.8 & 33.3 & 60.3 & 69 & 4.52 & .66 \\
\hline Evaluación & 0 & 6.3 & 17.5 & 25.4 & 50.8 & 69 & 4.2 & .95 \\
\hline Actividades gerenciales & 1.6 & 9.5 & 13.3 & 28.6 & 46 & 69 & 4.07 & 1.06 \\
\hline Coordinación & 0 & 6.3 & 12.7 & 20.6 & 60.3 & 69 & 4.34 & .93 \\
\hline Elaboración de proyectos & 1.6 & 7.9 & 15.9 & 17.5 & 57.1 & 69 & 4.20 & 1.08 \\
\hline Mediación & 0 & 9.5 & 9.5 & 25.4 & 55.6 & 69 & 4.26 & .98 \\
\hline Investigación & 1.6 & 6.3 & 14.3 & 28.6 & 49.2 & 69 & 4.17 & 1.00 \\
\hline Ejercicio de la docencia & 7.9 & 7.9 & 19 & 20.6 & 44.4 & 69 & 3.85 & 1.29 \\
\hline
\end{tabular}

Fuente: elaboración propia a partir de microdatos de la encuesta realizada en el proyecto de innovación "El perfil del trabajador social en la sociedad actual".

En tercer lugar, otras tareas que reciben respuestas muy favorables por parte de más del $50 \%$ de los entrevistados incluyen la prevención, elaboración de proyectos, promoción, mediación y evaluación. A este respecto, la desviación típica nos señala que las posiciones más extremas hacen referencia a la elaboración de los proyectos, mediación, evaluación, prevención y promoción. En cuarto lugar, las funciones que menor nivel de consenso, apuntan los encuestados se vinculan a la investigación, actividades gerenciales, ejercicio de la docencia y supervisión. Sin embargo, las mayores puntuaciones de desviación típica afectan al ejercicio de la docencia, actividades gerenciales, la investigación y la supervisión.

La mayoría de los encuestados destaca el desempeño de funciones que no se corresponden con su formación (55.6\%). Refieren administrativas (67.6\%), supervi- 
sión de actividades (17.6\%) y recursos humanos, planificación, evaluación y gestión económica (14.7\%) (Tabla 3). Los encuestados señalan que existen otros profesionales que desempeñan funciones propias de los trabajadores sociales (76.4\%).

Los acuerdos alcanzados con los tres grupos de discusión con profesionales están en concordancia con los resultados de la encuesta. Por un lado, trasladan como muy frecuentes la función de información y diagnóstico, apuntando tener exceso de funciones administrativas. Los participantes en los grupos de menores señalan que se debieran promover las funciones socio educativas de los profesionales del Trabajo Social.

TABLA 3: Funciones desempeñadas en el ámbito laboral y cualificación profesional

\begin{tabular}{|l|l|c|c|}
\hline & \multicolumn{1}{|c|}{ Respuestas } & \% & N \\
\hline Funciones que no se corresponden con & Sí & 55.6 & 35 \\
\cline { 2 - 4 } tu cualificación & No & 44.4 & 28 \\
\hline \multirow{2}{*}{$\begin{array}{l}\text { Tipos de funciones desempeñadas (sólo } \\
\text { a aquellos que responden que sí) }\end{array}$} & Administrativa & 67.6 & 23 \\
\cline { 2 - 4 } & $\begin{array}{l}\text { Recursos humanos, } \\
\text { planificación, } \\
\text { evaluación y gestión } \\
\text { económica }\end{array}$ & 14.7 & 5 \\
\cline { 2 - 4 } & $\begin{array}{l}\text { Supervisión de } \\
\text { actividades }\end{array}$ & 17.6 & \\
\hline & \multicolumn{2}{|c|}{6} \\
\hline $\begin{array}{l}\text { Funciones propias del Trabajo Social, } \\
\text { desempeñadas por otras profesiones }\end{array}$ & Sí & 74.6 & 47 \\
\cline { 2 - 4 } & No & 25.4 & 16 \\
\hline
\end{tabular}

Fuente: elaboración propia a partir de microdatos de la encuesta realizada en el proyecto de innovación "El perfil del trabajador social en la sociedad actual".

En lo referente al contenido expresado en el discurso entre el alumnado, se señaIan tres puntos claves para abordar el desarrollo de aspectos vinculados al desarrollo del método básico del Trabajo Social, el empleo de las herramientas de trabajo, tales como la realización de entrevistas o de encuestas y la distinción de los niveles de intervención (individual, grupal y comunitario). A estos aspectos, contribuyen las visitas a recursos y las charlas por parte de profesionales invitados a las asignaturas. Además, se valoran los aspectos jurídicos y modelos teóricos. En particular, los contenidos asociados a las leyes administrativas y el diseño tanto de proyectos como de los planes de intervención social. 


\subsection{Percepción profesional en torno a las funciones desempeñadas por los trabajadores sociales y a los ámbitos de intervención social}

Los mayores niveles de acuerdo en cuanto a profesiones sociales que desempeñan funciones del Trabajo Social hacen referencia a educadores sociales (50.8\%), integradores sociales $(32.3 \%)$ y psicólogos $(30.8 \%)$. Los mayores niveles de desacuerdo en los entrevistados hacen referencia a las profesiones educativas y de salud. A este respecto, el mayor grado de desacuerdo afectan a enfermeros (60.3\%), psicopedagogo (39.7\%) y animador sociocultural (34.9\%) El conjunto de las desviaciones típicas se sitúan entre 0.9 y 1.2

En los grupos de discusión, el intrusismo profesional aparece como un obstáculo y un elemento a considerar cuando se abordan las funciones profesionales vinculado, al igual que en la encuesta, a los psicólogos y educadores sociales.

TABLA 4: Señala ¿qué profesionales desempeñan las funciones propias del Trabajo Social y el nivel de acuerdo?

\begin{tabular}{|c|c|c|c|c|c|c|c|c|}
\hline & $\mathbf{1}$ & $\mathbf{2}$ & $\mathbf{3}$ & $\mathbf{4}$ & $\mathbf{5}$ & $\mathbf{N}$ & $\mathbf{M e d i a}$ & $\begin{array}{c}\text { Desviación } \\
\text { típica }\end{array}$ \\
\hline $\begin{array}{c}\text { Educador } \\
\text { social }\end{array}$ & 9.5 & 12.7 & 27 & 27 & 23.8 & 63 & 3.4 & 1.2 \\
\hline Psicopedagogo & 39.7 & 22.2 & 19 & 15.9 & 3.2 & 63 & 2.2 & 1.2 \\
\hline $\begin{array}{c}\text { Animador } \\
\text { Sociocultural }\end{array}$ & 34.9 & 31.7 & 19 & 11 & 3.2 & 63 & 2.1 & 1.1 \\
\hline Enfermero & 60.3 & 23.8 & 7.9 & 6.3 & 1.6 & 63 & 1.6 & 0.9 \\
\hline $\begin{array}{c}\text { Integrador } \\
\text { Social }\end{array}$ & 20.6 & 22.2 & 23.8 & 19 & 14.3 & 63 & 2.8 & 0.9 \\
\hline Psicólogo & 22.2 & 23.8 & 23.8 & 22.2 & 7.9 & 63 & 2.6 & 1.2 \\
\hline
\end{tabular}

Fuente: elaboración propia a partir de microdatos de la encuesta realizada en el proyecto de innovación "El perfil del trabajador social en la sociedad actual".

Se aprecian diferencias en la importancia que se concede a los ámbitos de intervención social tanto para el desarrollo, como para la consolidación del Trabajo Social. En orden de importancia, los datos recogidos en la tabla 5 señalan la mayor importancia en los ámbitos de servicios sociales, salud, justicia, educación, empleo, cooperación al desarrollo y ejercicio libre. 
TABLA 5: Percepción sobre la importancia y la consolidación del Trabajo Social por ámbitos y sectores de intervención social (1 menos importante y 5 más importante)

\begin{tabular}{|c|c|c|c|c|c|c|c|c|}
\hline & 1 & 2 & 3 & 4 & 5 & $\mathbf{N}$ & Media & $\begin{array}{c}\text { Desviación } \\
\text { típica }\end{array}$ \\
\hline \multicolumn{9}{|l|}{ IMPORTANCIA } \\
\hline Servicios Sociales & .0 & .0 & 6.3 & 9.5 & 84.1 & 63 & 4.77 & .55 \\
\hline Educación & .0 & 1.6 & 15.9 & 34.9 & 47.6 & 63 & 4.28 & .79 \\
\hline Salud & .0 & 1.6 & 14.3 & 33.3 & 50.8 & 63 & 4.33 & .78 \\
\hline Justicia & .0 & 3.2 & 14.3 & 30.2 & 52.4 & 63 & 4.31 & .83 \\
\hline Ejercicio libre & 3.2 & 14.3 & 36.5 & 28.6 & 17.5 & 63 & 3.42 & 1.04 \\
\hline Empleo & 1.6 & 11.1 & 20.6 & 30.2 & 36.5 & 63 & 3.88 & 1.07 \\
\hline $\begin{array}{c}\text { Cooperación al } \\
\text { desarrollo }\end{array}$ & 3.2 & 12.7 & 17.5 & 36.5 & 30.2 & 63 & 3.77 & 1.11 \\
\hline CONSOLIDACIÓN & 1 & 2 & 3 & 4 & 5 & $\mathbf{N}$ & Media & $\begin{array}{c}\text { Desviación } \\
\text { típica }\end{array}$ \\
\hline Servicios Sociales & 1.6 & 1.6 & 6.3 & 9.5 & 81.0 & 63 & 4.66 & .80 \\
\hline Educación & 6.3 & 11.1 & 19.0 & 33.3 & 30.2 & 63 & 3.69 & 1.19 \\
\hline Salud & 1.6 & 7.9 & 23.8 & 34.9 & 31.7 & 63 & 3.87 & 1.00 \\
\hline Justicia & 3.2 & 9.5 & 27 & 33.3 & 27 & 63 & 3.71 & 1.06 \\
\hline Ejercicio libre & 19 & 20.6 & 36.5 & 15.9 & 7.9 & 63 & 2.73 & 1.18 \\
\hline Empleo & 15.9 & 14.3 & 20.6 & 36.5 & 12.7 & 63 & 3.15 & 1.28 \\
\hline $\begin{array}{c}\text { Cooperación al } \\
\text { desarrollo }\end{array}$ & 9.5 & 14.3 & 23.8 & 38.1 & 14.3 & 63 & 3.33 & 1.17 \\
\hline
\end{tabular}

Fuente: elaboración propia a partir de microdatos de la encuesta realizada en el proyecto de innovación “El perfil del trabajador social en la sociedad actual"

Principalmente, este aspecto se aprecia en el mayor grado de importancia en la respuesta y la menor desviación típica. Al igual que en análisis anteriores, se observa un menor grado de dispersión en la respuesta. A este respecto, la percepción de los encuestados en torno a la consolidación sigue este mismo orden, a excepción de cooperación al desarrollo, que obtiene una puntuación mayor al ámbito del empleo.

Los profesionales consideran que las competencias que deben adquirir los estudiantes del grado de Trabajo Social son principalmente trabajo en equipo de carácter interdisciplinar $(81 \%)$, habilidades en las relaciones interpersonales $(79.4 \%)$ y resolución de problemas (71.4\%). Otras competencias son valoradas con un porcentaje inferior al $10 \%$ tales como adaptación a nuevas situaciones, resolución de problemas compromiso ético, capacidad de organización, planificación, reconocimiento de la diversidad y cuestiones burocráticas. 
TABLA 6: ¿Cuáles consideras que deben ser las competencias que deben adquirir los estudiantes en su proceso formativo?

\begin{tabular}{|l|c|c|}
\hline & \% multirespuesta & N \\
\hline Adaptaciones a nuevas situaciones & 7.9 & 5 \\
\hline Compromiso ético & 4.8 & 3 \\
\hline Capacidad de organización & 4.8 & 3 \\
\hline Planificación & 4.8 & 3 \\
\hline Habilidades en las relaciones interpersonales & 79.4 & 50 \\
\hline Resolución de problemas & 71.4 & 45 \\
\hline Trabajo en equipo de carácter interdisciplinar & 81.0 & 51 \\
\hline Formación & 0.0 & 0 \\
\hline Resolución de problemas & 7.9 & 5 \\
\hline Reconocimiento de la diversidad & 4.8 & 3 \\
\hline Temas burocráticos en la práctica existentes & 1.6 & 1 \\
\hline
\end{tabular}

Fuente: elaboración propia a partir de microdatos de la encuesta realizada en el proyecto de innovación “El perfil del trabajador social en la sociedad actual".

La tabla 7 recoge los resultados a la pregunta ¿Cuáles son los nuevos ámbitos de intervención del Trabajo Social? A esto los profesionales responden con un grado alto de dispersión. De todas las respuestas planteadas destacan de manera significativa dos. En primer lugar, los ámbitos relacionados con gestión, desarrollo y evaluación de proyectos $(23,81 \%)$ y en segundo lugar empresas, responsabilidad corporativa y ejercicio libre (19,05\%) En tercer lugar el ámbito de mediación y peritajes es seleccionado por $7,94 \%$ de los participantes. El resto de las opciones son seleccionadas por menos del $5 \%$ de encuestados.

Otra de las cuestiones que se recoge en el cuestionario hace referencia a las dificultades para un ejercicio profesional eficiente. Las respuestas se reflejan en la tabla 8.

Tal y como recoge la tabla 8 , cuando se pregunta a los encuestados acerca de las principales dificultades para un ejercicio profesional eficiente, las respuestas apuntadas serían en primer lugar la falta de recursos económico o humanos: recortes o falta de financiación $(17,46 \%)$, en segundo lugar, la burocracia, falta de comunicación y poder político $(15,87 \%)$, en tercer lugar las cuestiones de actualización de saberes y de información y el desconocimiento y falta de valoración social (ambas respuestas con un $12,7 \%$ ). Otra de las dificultades destacadas es el excesivo nivel de trabajo reflejado en el elevado ratio de profesionales $(11,11 \%)$. 


\section{TABLA 7: ¿Cuáles pueden ser los nuevos ámbitos de intervención del Trabajo Social?}

\begin{tabular}{|l|c|c|}
\hline & \% multirespuesta & N \\
\hline Ámbito de planificación & 3.17 & 2 \\
\hline Gestión, desarrollo y evaluación de proyectos & 281 & 15 \\
\hline Participación en la elaboración de proyectos de ámbito social & 3.17 & 2 \\
\hline Asociacionismo & 1.59 & 1 \\
\hline Cooperación entre entidades y administración pública & 1.59 & 1 \\
\hline Coordinación de equipos o de voluntariado y formador de equipos & 4.76 & 3 \\
\hline Mediación y peritajes & 7.94 & 5 \\
\hline Educación & 4.76 & 3 \\
\hline Empresas, responsabilidad social corporativa y ejercicio libre & 19.05 & 12 \\
\hline Género (educación afectivo sexual) & 3.17 & 2 \\
\hline Gerencia & 1.59 & 1 \\
\hline Planificación & 3.17 & 2 \\
\hline Intervención comunitaria & 3.17 & 2 \\
\hline Investigación & 4.76 & 3 \\
\hline E-Social Work & 1.59 & 1 \\
\hline Políticas de prevención & 3.17 & 2 \\
\hline No sé & 3.17 & 2 \\
\hline Vivienda & 1.59 & 1 \\
\hline
\end{tabular}

Fuente: elaboración propia a partir de microdatos de la encuesta realizada en el proyecto de innovación “El perfil del trabajador social en la sociedad actual".

En relación con las dificultades que se encuentran los profesionales en su "día a día", los integrantes de los tres grupos de discusión identificaron distintos obstáculos. Los principales elementos incluidos son la burocracia, la sobrecarga administrativa, la falta de formación especializada en su ámbito de intervención, la falta de trabajo en equipo y de trabajo en red por parte de los profesionales. Consideran, que todo ello obstaculiza el desempeño de una intervención social de calidad.

Unido a las dificultades, los participantes de los tres grupos a lo largo de las intervenciones plantean propuestas con la finalidad de mejorar la acción profesional y abordar las mismas. Incorporar el trabajo en equipo y el trabajo en red como una metodología exigible y que no quede al buen hacer de cada profesional; trabajar desde las fortalezas y las oportunidades en lugar de centrarse sólo en los problemas. Por último, se precisa incorporar acciones de inteligencia emocional y autocuidado para los profesionales.

Los obstáculos percibidos por los encuestados para la adquisición de competencias se recogen en la tabla 9. Consideran éstos que la principal dificultad se cen- 
tra en los planes de estudios formativos en la universidad y adecuación (profesorado: mucha teoría y falta de clases prácticas: tiempo de práctica. (28,6\%). Otro obstáculo que destaca es la falta de trabajo personal con cada profesional y escasa autocrítica $(4,8 \%)$.

\section{TABLA 8: ¿Cuáles crees que son las principales dificultades para un ejercicio profesional eficiente?}

\begin{tabular}{|l|c|c|}
\hline & \% multirespuesta & N \\
\hline $\begin{array}{l}\text { Actualización de saberes y de forma ción; falta de formación } \\
\text { práctica, saberes prácticos }\end{array}$ & 12.70 & 8 \\
\hline Autocomplacencia & 3.17 & 2 \\
\hline Burocracia, falta de comunicación y poder político & 15.87 & 10 \\
\hline $\begin{array}{l}\text { Falta de recursos económicos o humanos (recortes o problemas } \\
\text { de financiación) }\end{array}$ & 17.46 & 11 \\
\hline Dificultades en la coordinación de recursos & 3.17 & 2 \\
\hline Falta de tiempo & 3.17 & 2 \\
\hline Falta de trabajo en equipo por parte de los profesionales & 3.17 & 2 \\
\hline Excesivo nivel de trabajo (ratio de profesionales por usuario) & 11.11 & 7 \\
\hline Indefinición en competencias asignadas & 6.35 & 4 \\
\hline Falta de motivación (falta de empatía) & 4.76 & 3 \\
\hline Falta de poder decisorio & 1.59 & 1 \\
\hline Financiación del Tercer Sector & 1.59 & 1 \\
\hline $\begin{array}{l}\text { Falta de adecuación de las normativas o de nuevas leyes a las } \\
\text { necesidades de usuarios }\end{array}$ & 4.76 & 3 \\
\hline Desconocimiento y falta de valoración social & & 8 \\
\hline Precariedad laboral & 12.70 & 2 \\
\hline Falta de planificación & 3.17 & 1 \\
\hline
\end{tabular}

Fuente: elaboración propia a partir de microdatos de la encuesta realizada en el proyecto de innovación "El perfil del trabajador social en la sociedad actual." 


\section{TABLA 9: Obstáculos para la adquisición de competencias}

\begin{tabular}{|l|r|r|}
\hline Obstáculos en la adquisición de competencias & 1.6 & 1 \\
\hline Pérdida del nivel formativo del alumnado & 1.6 & 1 \\
\hline Investigación y publicación en áreas científicas & 3.2 & 2 \\
\hline Contexto de Trabajo & 1.6 & 1 \\
\hline Sociedad en general & 28.6 & 18 \\
\hline $\begin{array}{l}\text { Plan de estudios formativos en la universidad y adecuación (Profesorado; Mucha } \\
\text { teoría y falta de clases prácticas; Tiempo de prácticas) }\end{array}$ & 1.6 & 1 \\
\hline Dinámica de trabajo en las entidades & 4.8 & 3 \\
\hline Falta de trabajo personal con cada profesional y escasa autocrítica & 3.2 & 2 \\
\hline Formación en el desempeño profesional y reciclaje & 3.2 & 2 \\
\hline Falta de habilidades (inteligencia emocional o escucha) & 3.2 & 2 \\
\hline Falta de motivación & 3.2 & 2 \\
\hline Adecuación de prácticas académicas & 1.6 & 1 \\
\hline Habilidades técnicas (redacción de informes) & 1.6 & 1 \\
\hline Competencias éticas & 3.2 & 2 \\
\hline Desarrollo y madurez personal & 4.8 & 3 \\
\hline Otras & & \\
\hline
\end{tabular}

Fuente: elaboración propia a partir de microdatos de la encuesta realizada en el proyecto de innovación “El perfil del trabajador social en la sociedad actual".

\subsection{Reflexiones en torno al Grado de Trabajo Social. Visión de los estudiantes}

En los diferentes grupos de reflexión que se mantienen con los estudiantes del grado, estos trasladan sus apreciaciones acerca de las habilidades, estructura del grado, organización y horarios y otras cuestiones. Los resultados consensuados se exponen en la siguiente tabla. Al mismo tiempo, se puede señalar que los alumnos tienen una auto percepción alta en cuanto a la capacidad de aprendizaje en contenidos teóricos. Sin embargo, se aprecia una autopercepción baja en habilidades sociales. En concreto, estas referencias se vinculan al hecho de sentirse peor preparados en la atención directa de los usuarios, demandando mayor conocimiento en el "quehacer diario" y en la relación de acompañamiento. 


\section{TABLA 10: Reflexiones en torno al grado: habilidades, metodologías y estructura}

\begin{tabular}{|c|c|c|}
\hline \multirow[t]{3}{*}{$\begin{array}{l}\text { Habilidades y } \\
\text { metodologías } \\
\text { activas: }\end{array}$} & \multicolumn{2}{|c|}{$\begin{array}{l}\text { Habilidades adquiridas: } \\
\text { - } \quad \text { Trabajo en equipo, creatividad, pensamiento crítico, hablar en } \\
\text { público, colectivos vulnerables, proyectos, capacidades } \\
\text { comunicativas y compañerismo. }\end{array}$} \\
\hline & \multicolumn{2}{|c|}{$\begin{array}{l}\text { Metodología activa: } \\
\text { - } \quad \text { Prácticas, análisis de casos, visitas a instituciones. } \\
\text { - } \quad \text { Tutorización individual o tutorización entre iguales. }\end{array}$} \\
\hline & \multicolumn{2}{|c|}{ Rol del profesorado: cercanía, herramientas educativas. } \\
\hline \multirow[t]{6}{*}{$\begin{array}{l}\text { Estructura del } \\
\text { grado }\end{array}$} & \multirow[t]{2}{*}{$\begin{array}{l}\text { Organización de } \\
\text { horarios: }\end{array}$} & $\begin{array}{l}\text { Problemas: } \\
\text { - } \quad \text { Dispersión de horarios. }\end{array}$ \\
\hline & & $\begin{array}{l}\text { Propuestas: } \\
\text { - } \quad \text { Tiempo de descanso entre clases; } \\
\text { - } \quad \text { Mejor coordinación }\end{array}$ \\
\hline & \multirow[t]{2}{*}{$\begin{array}{l}\text { Planificación } \\
\text { curricular }\end{array}$} & $\begin{array}{l}\text { Problemas: } \\
\begin{array}{l}\text { - } \quad \text { Sobrecarga de estudiantes, concentración } \\
\text { de asignaturas y de trabajos }\end{array}\end{array}$ \\
\hline & & $\begin{array}{ll}\text { Propuestas: } \\
\text { - } \quad \text { Impartir asignaturas con un profesor y } \\
\text { - } \quad \text { Incorpar parcializar asignaturas; } \\
\quad 3^{\circ} \text { curso; } \\
\text { - } \quad \text { Vinculación de asignaturas troncales con el } \\
\text { objeto (estadística, derecho o economía) } \\
\text { del Trabajo Social. } \\
\text { - } \quad \text { Incluir asignaturas enfocadas al Trabajo } \\
\text { Social y Ciencias Sociales (Antropología, } \\
\text { Sociología y Psicología Social) }\end{array}$ \\
\hline & \multirow[t]{2}{*}{ Prácticas externas } & Problemas: Dispersión de horarios. \\
\hline & & $\begin{array}{ll}\text { Propuestas: } \\
\text { - } & \text { Realizar prácticas en distintas entidades o } \\
& \text { ámbitos; } \\
\text { - } & \text { Mejorar información de los procesos; } \\
\text { - } & \text { Flexibilizar las prácticas; } \\
\text { - } & \text { Incorporar aspectos relativos al } \\
& \text { conocimiento humano y la gestión de } \\
& \text { emociones. }\end{array}$ \\
\hline $\begin{array}{l}\text { Otras } \\
\text { consideraciones }\end{array}$ & \multicolumn{2}{|c|}{$\begin{array}{l}\text { Otros servicios de la universidad: Buena valoración de cursos, talleres, } \\
\text { becas e intercambios. }\end{array}$} \\
\hline
\end{tabular}

Fuente: elaboración propia a partir de los discursos del alumnado. 


\subsection{Conflictos éticos en la visión de los profesionales}

Atendiendo a la frecuencia con la cual se producen los dilemas éticos, se diferencian al menos cuatro problemáticas (Tabla 11). Se dan con mayor frecuencia hacen referencia al abuso de poder por parte de los superiores, el conflicto entre el secreto profesional y el deber de informar, las desavenencias con otros colegas respecto del mismo caso y el respeto de la autonomía de los usuarios. En segundo lugar, el ocultar parte de la verdad con la finalidad de no perjudicar al usuario se ha dado en el $41.3 \%$. El tercer lugar, las problemáticas vinculadas a la exigencia de contraprestación se producen con menor frecuencia son aquellas relacionadas con la duración o el tiempo que un expediente está abierto o de facilitar datos de contacto de un profesional (dirección o teléfono personal). En último lugar, los conflictos vinculados a las relaciones personales $(25.4 \%)$ y asistencia jurídica y revelación de datos confidenciales (17.5\%).

\section{TABLA 11: Principales dilemas que alguna vez has tenido en tu práctica profesional}

\begin{tabular}{|l|c|c|}
\hline & $\mathbf{\%}$ & $\mathbf{N}$ \\
\hline $\begin{array}{l}\text { Conflicto entre el cumplimie nto del secreto profesional y el deber de } \\
\text { informar }\end{array}$ & 57.1 & 63 \\
\hline Respeto a la autonomía de los usuarios & 54.0 & 63 \\
\hline Desavenencias con otros colegas respecto del mismo caso & 55.6 & 63 \\
\hline Relaciones personales con los usuarios & 25.4 & 63 \\
\hline Decir la verdad, no toda la verdad o mentira para no perjudicar a un usuario & 41.3 & 63 \\
\hline Exigencia de contraprestaciones al usuario/a como condición para acceder & 36.5 & 63 \\
\hline $\begin{array}{l}\text { Abuso de poder por parte de superiores y que afectan al adecuado } \\
\text { desarrollo }\end{array}$ & 58.7 & 63 \\
\hline Asistencia a juicios y revelación de datos confidenciales en esos contextos & 17.5 & 63 \\
\hline Duración del tiempo de intervención & 33.3 & 63 \\
\hline Facilitar datos de contacto del trabajador/a social & 30.2 & 63 \\
\hline
\end{tabular}

Fuente: elaboración propia a partir de microdatos de la encuesta realizada en el proyecto de innovación "El perfil del trabajador social en la sociedad actual."

Atendiendo al NC en las opciones de respuesta, pueden clasificarse las opiniones de los entrevistados que no han sido incluidas entre las 4 opciones preferentes por los entrevistados. A este respecto, se ha procedido a ordenar las respuestas en función a la tasa de respuestas en el ítem de NC (Tabla número 12).

En primer lugar, las respuestas con menor porcentaje en este ítem recogen aquellas que mayor importancia le dan los entrevistados. A este respecto, se identifican las desavenencias con otros colegas (14.3\%) y al conflicto entre el cumplimiento del secreto profesional y el deber de informar (23.8\%). En segundo lugar, 
alrededor del $20 \%$ de los entrevistados se decanta por el NC en las opciones vinculadas al respeto a la autonomía de los usuarios y al abuso de poder por parte de los superiores. En tercer lugar, en el rango entre el $30 \%$ y el $40 \%$ del NC se encuentran aquellas respuestas relativas a las relaciones personales con los usuarios, no decir la verdad para perjudicar al usuario, la duración del tiempo de intervención y la exigencia en las contraprestaciones. Por último, las mayores tasas de respuesta en la opción NC se asocian a facilitar los datos de contacto del Trabajador Social $(42.9 \%)$ y a la asistencia a juicios y revelación de datos confidenciales $(41.3 \%)$.

\section{TABLA 12: Dilemas éticos que consideras más importantes, ordenados por orden de importancia}

\begin{tabular}{|l|c|c|c|c|c|c|c|}
\hline & \multicolumn{2}{|c|}{ Respuestas a alguna de las opciones (\%) } & \multicolumn{3}{|c|}{ NC } \\
\hline & 1,0 & 2,0 & 3,0 & 4,0 & $\mathrm{~N}$ & $\mathrm{NC}$ & $\mathrm{N}$ \\
\hline $\begin{array}{l}\text { Conflicto entre el cumplimiento del } \\
\text { secreto profesional y el deber de } \\
\text { informar }\end{array}$ & 9.5 & 7.9 & 19 & 47.6 & 53 & 15.9 & 10 \\
\hline $\begin{array}{l}\text { Respeto a la autonomía de los } \\
\text { usuarios }\end{array}$ & 7.9 & 9.5 & 25.4 & 33.3 & 48 & 23.8 & 15 \\
\hline $\begin{array}{l}\text { Desavenencias con otros colegas } \\
\text { respecto del mismo caso }\end{array}$ & 12.7 & 31.7 & 27 & 14.3 & 52 & 14.3 & 9 \\
\hline $\begin{array}{l}\text { Relaciones personales con los } \\
\text { usuarios }\end{array}$ & 12.7 & 14.3 & 20.6 & 22.2 & 42 & 30.2 & 19 \\
\hline $\begin{array}{l}\text { IDecir la verdad, no toda la verdad o } \\
\text { mentir para no perjudicar a un } \\
\text { usuario }\end{array}$ & 9.5 & 15.9 & 23.8 & 19 & 43 & 31.7 & 20 \\
\hline $\begin{array}{l}\text { Exigencia de contraprestaciones al } \\
\text { usuario/a como condición para } \\
\text { acceder }\end{array}$ & 7.9 & 15.9 & 20.6 & 17.5 & 39 & 38.1 & 24 \\
\hline $\begin{array}{l}\text { Abuso de poder por parte de } \\
\text { superiores y que afectan al } \\
\text { adecuado desarrollo }\end{array}$ & 11.1 & 15.9 & 23.8 & 27 & 49 & 22 & 14 \\
\hline $\begin{array}{l}\text { Asistencia a juicios y revelación de } \\
\text { datos confidenciales en esos } \\
\text { contextos }\end{array}$ & 9.5 & 11.1 & 22.2 & 15.9 & 37 & 41.3 & 26 \\
\hline Duración del tiempo de intervención & 3.2 & 20.6 & 25.4 & 12.7 & 49 & 38.1 & 24 \\
\hline $\begin{array}{l}\text { Facilitar datos de contacto del } \\
\text { trabajador/a social }\end{array}$ & 7.9 & 9.5 & 9.5 & 30.2 & 36 & 42.9 & 27 \\
\hline Otros & 1.7 & 3.4 & 1.7 & 6.8 & 12 & 86.4 & 51 \\
\hline
\end{tabular}

Fuente: elaboración propia a partir de microdatos de la encuesta realizada en el proyecto de innovación "El perfil del trabajador social en la sociedad actual". 


\section{Conclusiones}

Este trabajo se enmarca, como se ha expuesto anteriormente en un Proyecto de Innovación Docente del área de Trabajo Social de la Universidad de La Rioja. Contribuye por un lado al análisis derivado de la reflexión de estudiantes y profesionales, y por otro a la concreción de propuestas que puedan mejorar los procesos formativos del grado. Incluso algunas de las cuestiones se podrían plantear como reformas del plan de estudios.

1. Se establecen las siguientes propuestas:

- Considerar las metodologías de aprendizaje significativo en la enseñanza.

- Seguir reforzando las habilidades grupales y comunicativas. Si bien, es necesario profundizar en habilidades vinculadas a la intervención social directa (entrevista, relación de ayuda o planteamiento de casos sociales).

- Incorporar los dilemas éticos en distintas asignaturas.

- Adaptar la presencia de asignaturas al objeto delTrabajo Social.

- Abordar la gestión de emociones, autoconocimiento y pensamiento crítico. Si bien, este aspecto es más necesario en las prácticas externas de la titulación puesto que es en ellas donde se manifiesta tanto la madurez como las carencias personales y relacionales del alumnado.

2. Las funciones profesionales delTrabajo Social son uno de los elementos clave que identifican a esta profesión. La información, diagnóstico, atención directa, planificación, fomento de la integración y coordinación son las tareas profesionales en las que existe un mayor consenso en su desempeño. Si bien, existen otras tareas tales como la gestión de proyectos o la investigación en las que no existe tanto consenso, siendo reconocidas en el Libro Blanco de la Aneca (2004) y en el estatuto de la profesión en Trabajo Social.

3. Un aspecto significativo de la práctica profesional es la realización de tareas que no se corresponden con la práctica profesional. En este grupo destacan fundamentalmente las administrativas, todos los profesionales se hacen eco de las implicaciones de la burocracia y la carga administrativa que esto conlleva.

En definitiva, este escenario apunta a mejorar la definición de los perfiles profesionales; tarea que es una responsabilidad compartida principalmente por Universidad, Colegios Profesionales, los propios trabajadores y trabajadoras sociales y también estudiantes del grado. 
La sociedad en general y las instituciones están demandando profesionales con competencias y habilidades tales como el trabajo en equipo, pensamiento crítico, creatividad, capacidades comunicativas, entre otras. Estas habilidades pueden adquirirse incorporando metodologías activas de enseñanza, con la finalidad de adquirir un aprendizaje significativo para los estudiantes. En esta línea los alumnos reclaman cambios y ajustes en los sistemas de enseñanza, que vayan más allá de las clases magistrales y exista una permeabilidad auténtica entre la realidad social y el ámbito universitario.

\section{Bibliografía}

Agrela Romero, B. Morales Villena, A. y Fuentes Gutiérrez, V. 2014 “Estudios de género en Trabajo Social: generando redes sobre docencia, investigación y metodologías en el aula.. Pp. 235-242 en El Trabajo Social ante el reto de la crisis y la educación superior, coordinado por E. Pastor Avilés, M.T. Martínez Fuentes, M. Avilés Hernández y Y. Doménech López. Madrid: Universitas.

Aguiar Fernández, G. 2006. "Análisis, desafíos y competencias desde el Trabajo Social". Acciones e investigaciones sociales, $n^{\circ}$ extraordinario 1.: 439-450. Consulta 21 de Mayo del 2019. (https://papiro.unizar.es/ojs/index.php/ais/article/view/4519).

ANECA. 2004. Libro Blanco. Título de Grado en Trabajo Social. Madrid: ANECA. Consulta 21 de Mayo del 2019. (http://www.aneca.es/var/media/150376/libroblanco_ trbjsocial_def.pdf)

Anguiano, A.M. y Plasencia Vázquez, C. 2007. "Formación de los Trabajadores Sociales: Competencias en la nivelación a la Licenciatura en Trabajo Social, módulo salud. Sistema abierto y a distancia." Margen: revista de Trabajo Social y Ciencias Sociales 44. Consulta el 05/06/2019. (https://www.margen.org/suscri/margen44/formac.html)

Barranco Expósito, G. 2011. "Conmemoración del 50 aniversario de los estudios de Trabajo Social en Tenerife (1960-2010)." Revista Canaria de Ciencias Sociales, nº 3,: 239-242.

Caparrós, N. y E. Raya, E. 2015. Métodos y técnicas de Investigación en Trabajo Social. 1 th ed. Madrid: Grupo 5..

Cea D'Ancona,, M.A. 2001. Metodología cuantitativa. Estrategias y técnicas de investigación social. 1th ed. Madrid: Síntexis.

Colom Masfret, D. 2004. "Estudios de la calidad percibida en los servicios de Trabajo Social de salud: un camino hacia la mejora." Trabajo Social y Salud, n 49: 39-60.

Colom Masfret, D. 2008. El Trabajo Social sanitario: Atención primaria y atención especializada, teoría y práctica. 1th ed. Madrid: Siglo XXI Editores.

Cortina, A. 2000. Ética de las profesiones. 1th ed. Estella: Verbo Divino.

Del Rincón, D. 2007. "Competencias docentes en la titulación de Trabajo Social." Humanismo y Trabajo Social, n6: 85-126

Expósito, G. 2011. “Conmemoración del 50 aniversario de los estudios de Trabajo Social en Tenerife (1960-2010)." Revista Canaria de Ciencias Sociales, n 3: 239-242.

Gil Parejo, M. 2004. "Los estudios de Trabajo Social y el proceso de convergencia europea en materia de educación superior." Trabajo Social Hoy, n 41: 29-42. 
Gómez Gómez, F. 2010. "Competencias profesionales en Trabajo Social." Portularia: Revista de Trabajo Social, vol. 10 (2), n 41, 2010: 51-63.

Gómez del Toro, R. y Fernández Sánchez, C. 2016. "Instrumentos de evaluación de competencias como recogida de evidencias de la consecución de resultados de aprendizaje en el Grado de Trabajo Social" en Transcender Bolonia a través de la innovación: más allá de un reto burocrático: una experiencia de innovación universitaria integrada, la Facultad de Ciencias Sociales de la UPO, Pp. 169-184. editado por G. Domínguez Fernández. Sevilla: UPO, 2016.

Gutiérrez Nieto, A. 2012. "Competencias y roles profesionales desde el Trabajo Social Sanitario en la continuidad asistencial." Trabajo Social y salud, n 72: 93-98.

Ibáñez, J. ed. 1985. Del algoritmo al sujeto. Perspectivas de la investigación social.Madrid: Editorial Siglo XXI.

Ituarte, A. 2009. "Trabajo social clínico en el contexto sanitario." Revista de Trabajo social y salud, $n^{\circ}$ 64: 283-304.

Leitao Ferreira, J.M. Álvarez Peña, P. Barroso Pena, M.J. 2018. "Situación actual y tendencias de los estudios de grado en trabajo social desde una perspectiva internacional". Pp. 47-68 en El trabajo social ante los desafíos del siglo XXI desde una perspectiva iberoamericana, editado por C. Verde Diego, A. Lima Fernández y E. Pastor SellerCizur el Menor: Thompson Reuters Aranzadi.

Martínez Navas, I. 2018. "Estudios de Trabajo Social en La Rioja (1972-2002)." Pp. 345388 en Ciencia y Esencia en la práctica del Trabajo Social, editado por E. Raya Díez, N. Caparrós Civera, B. Lorente Molina, y S. Anaut Bravo, S. Valencia: Tiranch Lo Blanch. Colección de Economía y de Sociología.

Martínez- Román, M.A., Doménech López, Y. Domínguez Alonso, F. Escartín Caparrós, M.J. Giménez Bartomeu, M. Mohedano, R. Lilló Bebeyto, A. y Suárez Soto, E. 2013. “Estudio transversal de las asignaturas "Trabajo Social con Personas y Familias, Trabajo Social con Grupos y Trabajo Social con Comunidades." Pp. 1286-1299 en Diseño de acciones de investigaciones en docencia universitaria, editado por J.D. Álvarez Teruel, M.T. Tortosa Ibáñez y N. Pellín Buades. Alicante: Universidad d'Alicante. Servicio de Publicaciones.

Medina Ruiz, E. y Blanco Baños, G. 2014. "Evaluación de las competencias del título de trabajo social desde la perspectiva de los estudiantes". Pp. 1286-1299, en El trabajo social ante el reto de la crisis y la educación superior, editado por E. Pastor Seller, M.T. Martínez Fuentes, M. Avilés Hernández y Y. Doménech López. Madrid: Universitas.

Ramos-Feijóo, C. Ariño, M., Berasaluze, A. Dellavalle, M. Lorenzo, J. y Vaílo, P. 2013. “ Sinergias interuniversitarias en las estrategias didácticas para mejorar la adquisición de competencias en Trabajo Social." Pp. 1233-1242, en XI Jornadas de redes de investigación en Docencia Universitaria: Retos de futuro en la enseñanza superior: docencia e investigación para alcanzar la excelencia académica, editado por J.D. de Álvarez Teruel, M.T. Tortosa Ibáñez, M y N. Pellín Buades. Alicante: Universidad de Alicante. Instituto de Ciencias de la Educación.

Ramos Feijóo, C. Lorenzo García, J. y Valílo Pertusa, P. 2013. “La percepción del alumnado sobre la adquisición de competencias en el Grado de Trabajo Social." Pp. 2281-2296, en XI Jornadas de redes de investigación en Docencia Universitaria: Retos de futuro en la enseñanza superior: docencia e investigación para alcanzar la excelencia académica, edi- 
tado por J.D. Álvarez Teruel, J.D., M.T. Tortosa Ybáñez, y N. Pellín Buades, N., Alicante: Universidad de Alicante. Instituto de Ciencias de la Educación.

Raya, E., Caparrós, N. y Carbonero, D. 2016. “Nuevos escenarios de intervención en trabajo social para la innovación." Pp. 117-128, en Emprendimiento, innovación y RSC en trabajo social. Madrid: Grupo 5.

Ruiz Rodríguez, M.P. 2013. “El Trabajo Social como perito, testigo y especialista del sistema judicial español". Documentos de Trabajo. consultado el 05 de Junio de 2019, (https://es.scribd.com/document/136184050/El-trabajador-social-forense-como-perito-testigo-y-especialista-del-sistema-judicial-espanol)

Méndez Francisco, L. 1987. "Propuesta para una renovación del plan de estudios de Trabajo Social." Cuadernos de Trabajo Social n 0,: 45-72.

Monreal Gimeno, C. y Terrón-Caro, M.T. 2012. "Una experiencia interdisciplinar y evaluación de competencias en la doble titulación de Trabajo Social y Educación Social." UPO INNOVA: revista de innovación docente: 350-368. Consulta el 4 de mayo de 2020. (https://revistas/index.php/upoinnova/article/view/112.pdf)

Raya Díez, E. y Caparrós Civera, N. 2013. “Trabajo social en las relaciones laborales y la empresa: vías para el emprendimiento". Documentos de Trabajo Social, n52: 338-356.

Restrepo Giraldo, F.J. 2013. "Epistemología del Trabajo Social." Trabajo Social (Universidad Nacional de Colombia), $n^{\circ}$ 4: 23-30. Consulta el 5 de abril de 2020. (https://revistas.unal.edu.co/index.php/tsocial/article/view/32512.pdf).

Sabater Fernández, C. 2015. "La muestra en la investigación cuantitativa". Pp. 95-122 en Métodos y técnicas de Investigación en Trabajo Social, editado por N. Caparrós, y E. Raya. Madrid: Grupo 5.

Secretaría de Instituciones Penitenciarias. 2018. "Manual de Procedimiento de Trabajo Social en Instituciones Penitenciarias. Madrid: Ministerio del Interior (Secretaría de Instituciones Penitenciarias). Documentos de Trabajo. Consulta 05 de junio de 2019, ( http://www.institucionpenitenciaria.es/web/export/sites/default/datos/descargables/estad pm/Manual_Trabajo_Social_09_04_2018.pdf)

Serrano-Martínez, C. Caparrós Civera, N. Carbonero Muñoz, D. y Cuesta Ruiz Clavijo, A. 2019. "Aprendizajes y competencias en el Grado de Trabajo Social. Reflexiones del alumnado hacia su profesionalización." Revista Interacción y Perspectiva, Vol. 9(1): 10-24. Consulta el 05 de abril de 2019. (http://produccioncientificaluz.org/index.php/interaccion/article/view/29834)

Vázquez Aguado, O. 2002. "Trabajo Social y competencia intercultural." Portularia: Revista de Trabajo Social, Vol. 2: 125-138.

Vázquez Aguado, O. 2011. “Las competencias profesionales en los títulos de grado en trabajo social." Servicios sociales y política social, 96: 2011: 21-36.

Ruiz de Olabuénaga, J.I. ed. 1989. Metodología de investigación cualitativa. Colección de Sociología. Bilbao: Universidad de Deusto.

Zamanillo, T. y Gaitán, L. 1991. Para comprender el Trabajo Social. Estella (Navarra): Verbo Divino. 


\section{ARTICULOS/ARTICLES}

El sentido de comunidad en la adaptación de los inmigrantes latinoamericanos / Sense of community in the adaptation of Latin American immigrants

Mario Millán-Franco, Luis Gómez-Jacinto, María Isabel Hombrados-Mendieta y Gloria Kirwan

Correlation between emotional intelligence and problem-solving skills of greek social work students / Estudio de correlación entre la inteligencia emocional y las habilidades de resolución de problemas de los estudiantes griegos de trabajo social

Charis Asimopoulos, Sophia Martinaki y Aggeliki Papaioannou

La necesaria simbiosis entre el Trabajo Social y el desarrollo regional: formación y perfil profesional para la intervención social en el territorio / The necessary symbiosis between Social Work and regional development: training and professional profile for social intervention in the territory

Isaac Enríquez Pérez

La huella generacional del magisterio femenino en Extremadura (1958-2018) / The generational footprint of the female teachers in Extremadura (1958-2018)

Santiago Cambero Cambero Rivero y Marta García Romero

Págs 81-107

Competencias y funciones profesionales: un análisis aplicado a la titulación de trabajo social en la universidad de la Rioja / Professional competences and functions: an analysis applied to the social work degree at the university of la Rioja

Domingo Carbonero Muñoz, Ana Belén Cuesta Ruiz Clavijo y Neus Caparrós Civera, Cecilia Serrano-Martinez

\section{BESENIAS/REVIEWS}

Rings, G., Rasinger, S. 2020. The Cambrigde Handbook of Intercultural Communication / El Cambridge Handbook sobre Comunicación Intercultural. Cambridge: Cambridge University Press 